\title{
Carpal Kinematics following Sequential Scapholunate Ligament Sectioning
}

\author{
Clare E. Padmore, MEng ${ }^{1,2}$ Helen Stoesser, MESc ${ }^{1,2}$ \\ James A. Johnson, $\mathrm{PhD}^{1,2}$ Nina Suh, MD, FRCSC ${ }^{1,2}$ \\ 1 Bioengineering Research Laboratory, Roth McFarlane Hand and Upper \\ Limb Centre, St. Joseph's Health Care London, London, Ontario, Canada \\ 2 The University of Western Ontario, London, Ontario, Canada
}

J Wrist Surg 2019;8:124-131.
G. Daniel G. Langohr, $\mathrm{PhD}^{1,2}$

Address for correspondence Nina Suh, MD, FRCSC, Bioengineering Research Laboratory, Roth McFarlane Hand and Upper Limb Centre, St. Joseph's Health Care London, 268 Grosvenor St., London, Ontario N6A 4L9, Canada (e-mail: Nina.Suh@sjhc.london.on.ca).

\begin{abstract}
Background The scapholunate ligament (SLL) is the most commonly injured intercarpal ligament of the wrist. It is the primary stabilizer of the scapholunate (SL) joint, but the scaphotrapeziotrapezoid (STT) and radioscaphocapitate (RSC) ligaments may also contribute to SL stability. The contributions of SL joint stabilizers have been reported previously; however, this study aims to examine their contributions to SL stability using a different methodology than previous studies.

Purpose The purpose of this in vitro biomechanical study was to quantify changes in SL kinematics during wrist flexion and extension following a previously untested sequential sectioning series of the SL ligament and secondary stabilizers.

Methods Eight cadaveric upper extremities underwent active wrist flexion and extension in a custom motion wrist simulator. SL kinematics were captured with respect to the distal radius. A five-stage sequential sectioning protocol was performed, with data analyzed from 45-degree wrist flexion to 45-degree wrist extension.

Results Wrist flexion and extension caused the lunate to adopt a more extended posture following sectioning of the SLL and secondary stabilizers compared with the intact state $(p<0.009)$. The isolated disruption to the dorsal portion of the SLL did not result in significant change in lunate kinematics compared with the intact state $(p>0.05)$. Scaphoid kinematics were altered in wrist flexion following sequential sectioning $(p=0.013)$. Additionally, disruption of the primary and secondary stabilizers caused significant change to SL motion in both wrist flexion and wrist extension $(p<0.03)$.

Keywords

- scapholunate instability

- wrist

- biomechanics

Conclusions The SLL is the primary stabilizer of the SL articulation, with the STT and RSC ligaments playing secondary stabilization roles.

Clinical Relevance Understanding the role primary and secondary SL joint stabilizers may assist in the development of more effective treatment strategies and patient outcomes following SLL injuries.
\end{abstract}

The wrist joint is composed of a complex configuration of ligaments connecting adjacent carpal bones, allowing for a

All work was performed at the Hand and Upper Limb Clinic, London, Ontario. mobile carpus. ${ }^{1,2}$ Ligamentous injuries have the potential to disrupt the delicate balance within the wrist joint, leading to altered carpal kinematics, abnormal joint loading, and secondary degenerative changes. ${ }^{2}$ The most commonly injured intercarpal ligament is the scapholunate ligament $(\mathrm{SLL})(\mathbf{- F i g} \cdot \mathbf{1})^{2-5}$ received

February 7, 2018

accepted after revision

November 12, 2018

published online

January 17, 2019
Copyright (c) 2019 by Thieme Medical

Publishers, Inc., 333 Seventh Avenue, New York, NY 10001, USA Tel: +1(212) 584-4662
DOI https://doi.org/ $10.1055 / \mathrm{s}-0038-1676865$. ISSN 2163-3916. 


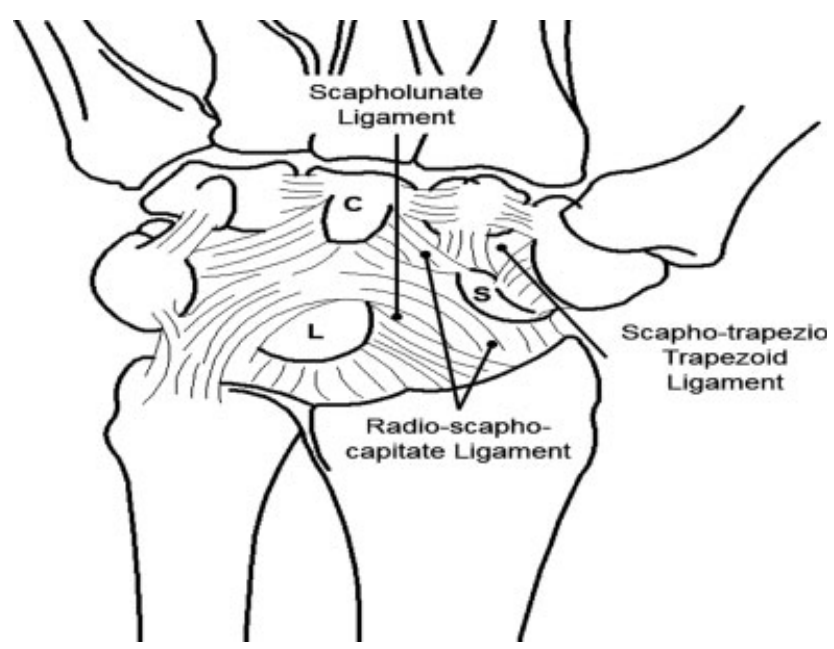

Fig. 1 A volar view of the ligaments sectioned during the testing protocol, including the scapholunate ligament and secondary stabilizers.

Oftentimes, initial radiographs are normal, and acute injuries may be missed. The scaphoid and lunate dissociate following more severe SLL injuries and eventually the scaphoid assumes a flexed position while the lunate rotates into extension. This results in dynamic and then eventual static carpal malalignment. ${ }^{4,6}$ Ultimately, a predictable pattern of degenerative changes occurs at the radioscaphoid joint, referred to as scapholunate (SL) advanced collapse. ${ }^{7}$

The SLL is considered the primary ligamentous restraint of the scaphoid and lunate. ${ }^{2,8}$ It has three anatomical regions (dorsal, proximal, and volar) of different material and anatomical properties, whereby the dorsal portion is the thickest and most critical of the SL stabilizers. ${ }^{2,9,10}$ However, there are two additional ligaments that may be secondary stabilizers of the SL joint: the radioscaphocapitate (RSC) ligament and the scaphotrapeziotrapezoid (STT) ligament ${ }^{8}$ (-Fig. 1). Although the ligamentous anatomy of these structures is well documented, further study is required to investigate isolated SL injuries.

Numerous biomechanical studies have evaluated the effect of sectioning the SL ligament and the secondary stabilizers. $^{2,11-15}$ These studies examined varying motion pathways and ligament-sectioning sequences to determine the role of the primary SL stabilizers and secondary stabilizers; however, due to the number of ligaments that help to stabilize the SL articulation, there remain sectioning sequences that have gone unexamined. Although previous studies have provided insight into the contributors of SL stability, most studies were limited by a truncated range of motion.

Clinically, there remains a paucity of evidence as to which stabilizers should be repaired and/or reconstructed following SLL injuries. As the contributors of SL instability remains unclear, current treatment strategies vary and often result in poor or unpredictable long-term clinical outcomes for patients. ${ }^{12,14,16-23}$

The purpose of this in vitro biomechanical study was to quantify changes in SL kinematics during wrist flexion and extension following a previously unexamined sequential sectioning protocol, which included simulation of isolated SLL injuries and injury to secondary stabilizers, using an active motion simulator.

\section{Material and Methods}

\section{Specimen Preparation}

Eight fresh-frozen cadaveric upper limbs (mean age: 69 years [range: 54-85]; 8 males; 8 right) amputated mid-humerus were used in this study. Computed tomography (CT) scans of each wrist were examined prior to inclusion in the study to rule out any underlying wrist pathology. SLL, STT, and RSC integrity and location was confirmed through fluoroscopy and subsequently by direct visualization during the insertion of the optical tracking mounts through a ligament-sparing dorsal wrist capsulotomy to preserve the dorsal intercarpal and dorsal radiocarpal ligaments. The SL angle was measured during fluoroscopic visualization to ensure it was within normal margins. Each upper limb specimen was thawed prior to testing for 18 hours at room temperature, and all soft tissue structures were left intact.

Optical tracking markers (Optotrak Certus, Northern Digital, Waterloo, Ontario, Canada) were secured to the lunate, scaphoid, third metacarpal, ulna, and radius to capture the three-dimensional motion of each structure during testing. Two cortical bone screws (Ø $2.7 \mathrm{~mm}$ ) were used to attach optical trackers to both the scaphoid and lunate and were inserted under fluoroscopic guidance to ensure secure tracker fixation. Lunate screws were inserted through a dorsal midline incision centered on the lunate body aimed toward the volar rim, and scaphoid screws were inserted through a volar incision over the tuberosity. Each specimen was subjected to a full range of wrist motion under fluoroscopic control to ensure that there was no screw or tracker impingement. The optical trackers were oriented to maintain an optimal line of sight during testing. The third metacarpal tracker was inserted through a dorsal incision on the distal diaphysis, the ulnar tracker was inserted into the proximal one-third of the shaft, and the radial tracker was secured into

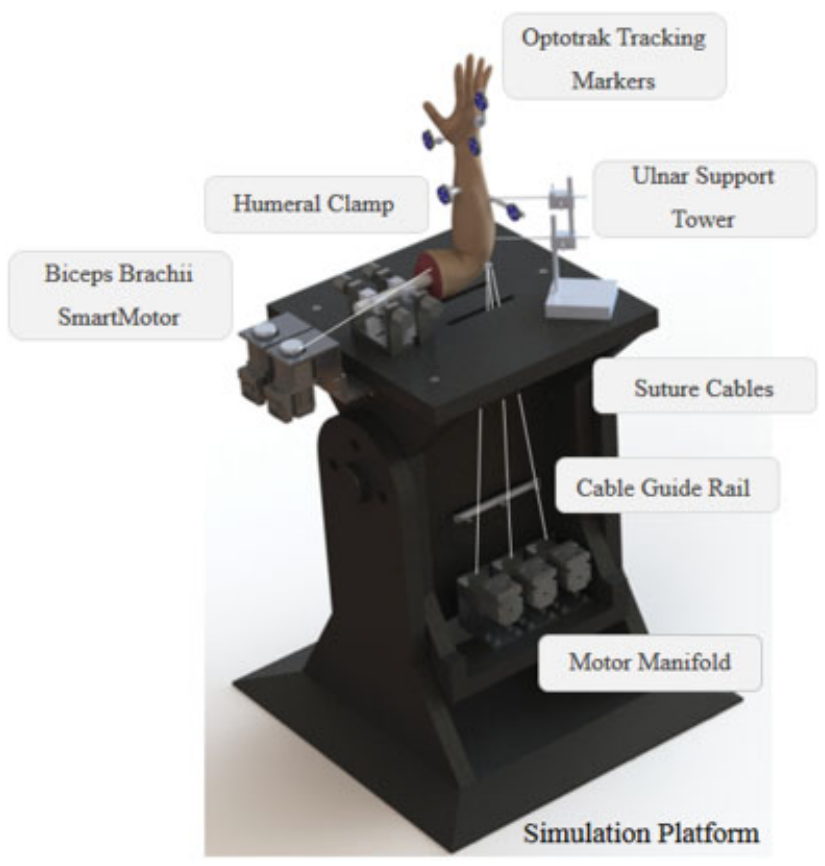

Fig. 2 In vitro active motion simulator capable of loading the seven muscle groups of interest to simulate active wrist flexion and extension. 
the distal two-thirds of the shaft using cortical bone screws (Ø $3.5 \mathrm{~mm}$ ).

The flexor and extensor tendons of the wrist including the extensor carpi radialis brevis, extensor carpi radialis longus, extensor carpi ulnaris, flexor carpi radialis, and flexor carpi ulnaris were exposed and sutured at the musculotendinous junction to facilitate the application of muscle forces (Ethicon, Somerville, NJ). The biceps brachii and pronator teres (PT) were also sutured to permit the control of forearm rotation. Epicondyle blocks were fixed to the lateral and medial epicondyles of the humerus to guide the suture lines and maintain the physiological line of action of the tendons. The specimens were then mounted on a custom wrist motion simulator capable of producing active wrist motion by rigidly securing the humerus using a clamp ${ }^{24}$ (-Fig. 2). Two threaded pins were used to fix the shaft of the ulna to the support tower aligning the elbow at 90-degree flexion. The suture lines were linked to their corresponding SmartMotor (SM2316D-PLS2, SMI Animatics Corp.) at the base of the simulator platform. The digits of each specimen were manipulated into full flexion and immobilized using Coban Self-Adherent Wrap (3M, Elyria, OH).

\section{Testing Protocol}

Prior to testing, anatomical landmarks on the radius and third metacarpal, which were palpated, were digitized once the specimen was mounted to the active motion wrist simulator to create relevant coordinate systems. Neutral position of the wrist was defined as alignment of the third metacarpal with the long axis of the radius, and this angle was used to define wrist position. The generated coordinate systems served to calculate the angle of wrist flexion and extension throughout motion. ${ }^{25}$ To maintain neutral forearm pronation and supination, which was defined as the thumb parallel to the humerus, the biceps brachii and PT were held at a constant position during motion trials. ${ }^{25}$ Due to the PT and biceps brachii having different moment arms, on average, the force in the biceps was greater than the force exerted by PT.

An intact cyclic motion trial of planar wrist flexion and extension (45-degree flexion to 45-degree extension) was initially performed. Out-of-plane motion was minimized ( $3 \pm 3$ degrees) with the wrist motion simulator's closedloop feedback control system. Subsequently, a four-stage sectioning protocol (S1: intact; S2: dorsal SL cut; S3: SL cut; S4: SL and STT cut; S5: SL, STT, and RSC cut) was performed using a no. 15 surgical scalpel blade under direct visualization. The midportion of the lunate in the sagittal plane was used as the anatomical landmark to bisect the volar and dorsal SLL. As a result, the dorsal SLL sectioning encompassed the SLL from the midpoint of the lunate on the sagittal plane to its dorsal edge. Conversely, the volar SLL was sectioned from the midpoint of the lunate to its volar edge. After each stage of sectioning, a freer was inserted to ensure full release of the intended ligament. Motion trials were performed following each stage of the protocol. Motion trials were performed at $\sim 5$ degrees per second, and two motion paths were executed including wrist flexion (0-45 degrees) and extension (0-45 degrees).
Kinematic data were collected continuously at 4,600 Hz; however, to avoid oversampling, the dataset was reduced, and results were reported in 5-degree increments for the two aforementioned motion paths. The global coordinate system was defined using the Optotrak camera, and all measurements were with respect to the camera. The skin was closed throughout each stage of the testing protocol to maintain specimen hydration. Following testing, each specimen was denuded and the joints were disarticulated. Anatomical landmarks on the bones of interest (the radius, third metacarpal, scaphoid, and lunate) were digitized using a pointed stylus to generate clinically relevant coordinate systems on the bony anatomy. Output data from the Certus Optotrak was then transformed from the optical tracking markers to anatomical coordinate systems.

\section{Outcome Variables and Data Analysis}

To quantify the effects of sequential sectioning on carpal kinematics, we evaluated two motion paths: wrist flexion and wrist extension. The wrist flexion motion path was defined as the specimen starting in neutral wrist position and progressing to 45 degrees of wrist flexion. The wrist extension motion path was defined as the specimen starting in neutral wrist position and progressing to 45 degrees of wrist extension.

All joint angles were calculated using the relative orientation of each bone's coordinate system, which were determined through transformations of the recorded tracker position. The rotation of the scaphoid and lunate relative to the distal radius was evaluated for both motion paths and for each of the five different stages of sectioning. The mean angular difference in carpal bone rotation in the flexion-extension plane between each stage of testing was calculated for both motion paths. Meanwhile, SL intercarpal motion was calculated using custom software, which provided the relative rotation of the scaphoid with respect to the lunate in the flexion-extension plane.

During the denuding process, the success of SLL, STT, and RSC sectioning was gauged and specimens that had been incompletely sectioned were not included in the results. A total of three specimens were not included in the final data analysis. Had these specimens been included, the sample size would have increased to 11 .

\section{Statistical Methods}

To detect statistical differences in scaphoid, lunate, and SL rotation between the intact state and the sectioning stages (S1: intact; S2: dorsal SL cut; S3: SL cut; S4: SL and STT cut; S5: SL, STT, and RSC cut), three three-way repeated-measures analysis of variance (RM-ANOVA) were performed using SPSS 17.0 (SPSS Inc., Chicago, IL), with Bonferroni correction factor for multiple comparisons. The factors included flexion and extension, sectioning stage (S1: intact; S2: dorsal SL cut; S3: SL cut; S4: SL and STT cut; S5: SL, STT, and RSC cut), and wrist angle (5-degree increments). These RM-ANOVA tests were used to determine differences in carpal kinematics between flexion and extension and the sectioning stages. Main effects as well as posthoc pairwise comparisons are reported for the outcome variables. A posthoc power study demonstrated that a sample size of eight specimens was sufficient to have greater than $80 \%$ 


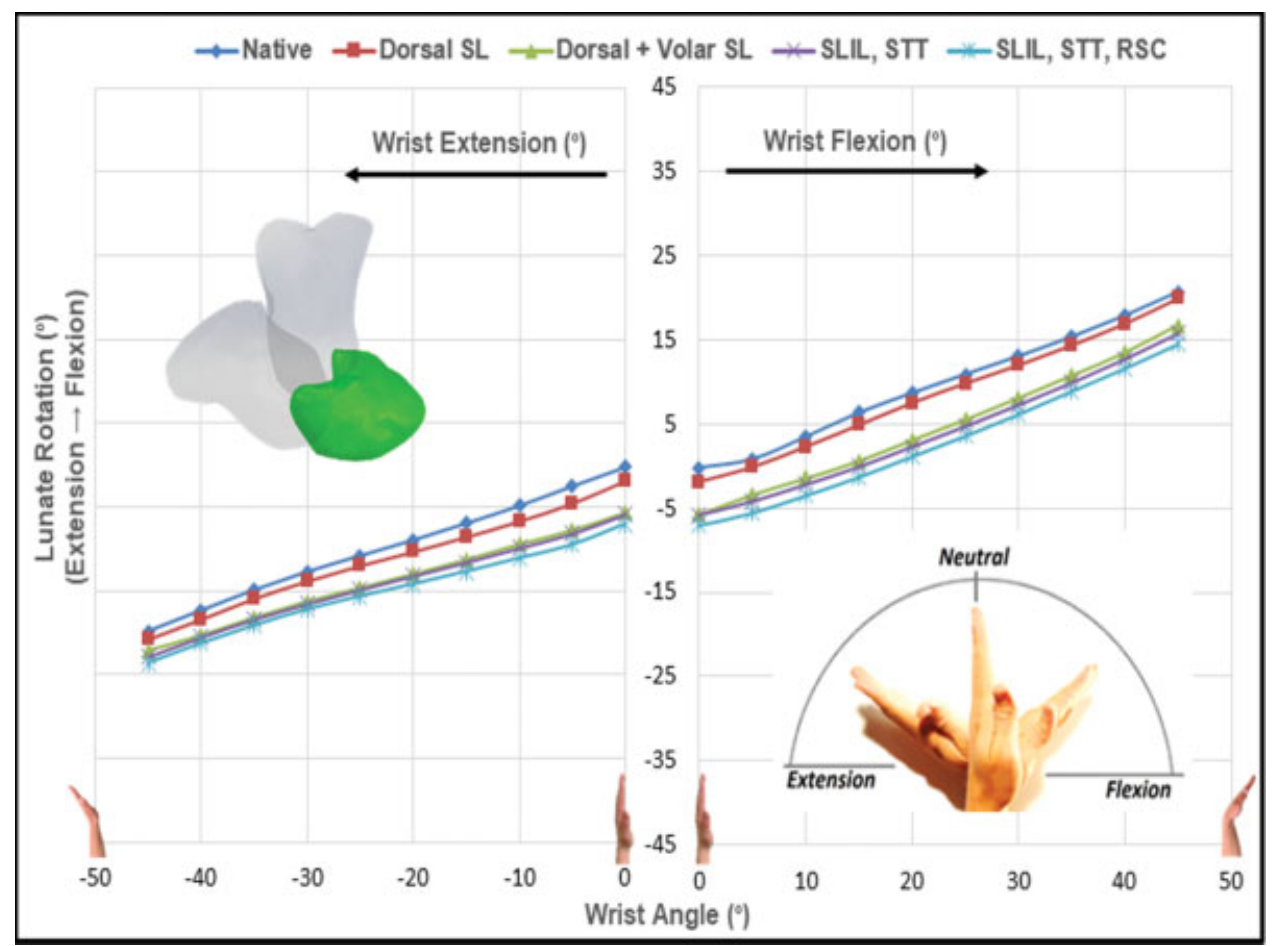

Fig. 3 Mean lunate motion in the flexion-extension plane for the flexion and extension motion paths. The graph illustrates the lunate's progression to a more extended position during wrist flexion and extension as more stabilizing structures are sectioned. Standard deviations were omitted for clarity (S1: 1.2-11.5; S2: 2.3-13.5; S3: 3.1-14.5; S4: 3-14; S5: 2.7-14).

power, with $95 \%$ confidence for each comparison that was tested. Statistical significance was set at $p<0.05$.

\section{Results}

\section{Lunate Kinematics}

During the flexion motion path, the lunate was observed to adopt a more extended posture following complete sectioning of the primary and secondary stabilizers compared with the intact state when examining the main effects ( $\mathbf{- F i g} . \mathbf{3}$; $p=0.001$ ). The largest increase in lunate extension was observed at 30 degrees of wrist flexion for all four sectioning states when compared with the intact state ( $6.4 \pm 4$ degrees). The isolated disruption to the dorsal portion of the SLL did not result in significant extension of the lunate compared with the intact state (S2: $p=1)$. Although the lunate adopted a more extended position across the wrist flexion motion arc after complete SLL disruption, it was not statistically different from the intact state (S3: $p=0.054)$. Conversely, a posthoc pairwise comparison detected a significant extension of the lunate following sectioning the secondary stabilizing ligaments compared with the intact state across the wrist flexion motion arc (S4: $p=0.03 ; \mathrm{S} 5: p=0.017$ ).

During the wrist extension motion path, the lunate also adopted a more extended posture following the sectioning of the primary and secondary stabilizers compared with the intact state $(p=0.009)$. However, lunate posture was not significantly different than the intact state following the isolate disruption of the dorsal SLL (S2: $p=0.23$ ). Further sectioning resulted in not only significantly more lunate extension compared with the intact state but also significantly more exten- sion with each progressive stage apart from the progression from complete SLL tear to sectioning of the STT ligament (S1S3: $p=0.039$; S1-S4: $p=0.02$; S1-S5: $p=0.013$; S2-S3: $p=0.019 ;$ S3-S4: $p=0.18$; S4-S5: $p=0.038$ ).

\section{Scaphoid Kinematics}

During wrist flexion, when all sectioning states were generally compared, scaphoid flexion increased ( - Fig. $4 ; p=0.013$ ). The largest increase in scaphoid flexion was observed at 20 degrees of wrist flexion for all four sectioning states when compared with the intact state (of $4.8 \pm 3.5$ degrees). However, the pairwise comparison of scaphoid motion with the sectioning stages was not significant ( - Table 1). Additionally, scaphoid motion was not significantly different during wrist extension $(p=0.71)$.

\section{Scapholunate Intercarpal Kinematics}

During wrist flexion, SL motion was significantly different between the sectioning stages ( $\boldsymbol{- F i g . ~ 5 ; ~} p=0.003$ ). The largest change in SL motion was observed at 20 degrees of wrist flexion for all four sectioning states when compared with the intact state ( $10 \pm 6$ degrees). Following the isolated disruption of the dorsal SLL, there was little change in SL motion compared the intact state ( $2: p=0.35$ ). Further progression through the sectioning stages resulted in significantly more SL intercarpal motion compared with the intact state (S3: $p=0.028$; S4: $p=0.044$; S5: $p=0.033$ ). During wrist extension, SL motion was significantly different between the sectioning stages $(p=0.032)$. However, the pairwise comparison of SL intercarpal motion with the sectioning stages was nonsignificant (-Table1). 


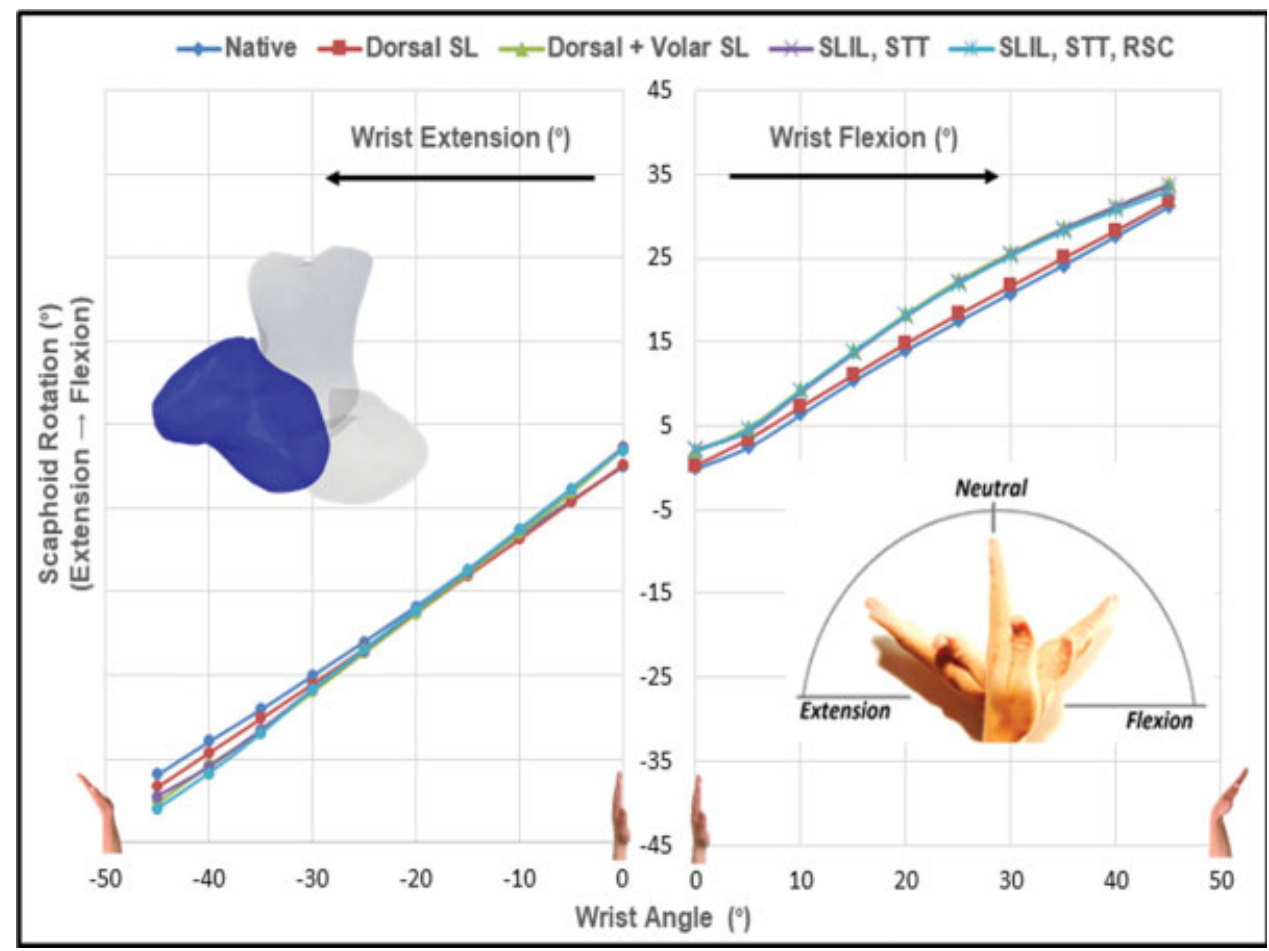

Fig. 4 Mean scaphoid motion in the flexion-extension plane for the flexion and extension motion paths. Sequential sectioning caused the scaphoid to adopt slightly more flexed posture in wrist flexion. Standard deviations were omitted for clarity (S1: 0.79-4.6; S2: 1.9-5.6; S3: $2.7-$ 6.2; S4: 2.9-7; S5: 3.5-6.5).

\section{Discussion}

This study provides insight into the stabilizing function of the SLL, STT, and RSC ligaments. Scaphoid, lunate, and SL intercarpal motion was captured after sequential sectioning and compared with the intact state. The results support previous literature, which suggests that the SLL plays a critical role in maintaining SL stability; however, isolated dorsal SLL injuries alone do not cause significant alteration in scaphoid or lunate kinematics. Furthermore, secondary ligamentous restraints were also found to significantly contribute to the maintenance of normal SL kinematics. The lunate was observed to adopt a significantly more extended posture as more stabilizing structures were sectioned. Interestingly, during wrist extension, the lunate adopted a less extended posture relative to the distal radius compared with wrist flexion following sequential sectioning. Contrasting previous studies by that of Short et al, we noted little change in scaphoid kinematics during wrist extension. Our results do have some similarities to the findings of Short et al, who also reported that alterations to carpal kinematics were more apparent in wrist flexion. It is perhaps not surprising that due to the volar location of the STT, RSC, and volar SL ligaments, sectioning of these structures had a greater stabilizing function in wrist flexion.

Isolated sectioning of the dorsal portion of the SLL induced small nonsignificant changes in SL angular rotation. Additionally, SL motion remained relatively unchanged with the isolated sectioning of the dorsal portion of the SLL. These results contrast several previous biomechanical and histological stu- dies, which reported that the dorsal component of the SLL plays a critical role in SL joint stability. ${ }^{9,10,15}$ Waters et $\mathrm{al}^{15}$ evaluated the stabilizing and functional role of the dorsal and volar portions of the SLL with respect to SL kinematics and found that the dorsal portion of the SLL induces larger angular changes when sectioned compared with the volar portion. Berger et $\mathrm{al}^{10}$ investigated material properties of the different anatomical regions of the SLL and concluded that the failure force of the dorsal SLL was more than twice the failure force of the volar SLL and four times the force of the proximal SLL. Although we did not investigate the role of sectioning order, this study did not demonstrate significant angular changes following sectioning of the dorsal portion of the SLL.

Interestingly, following the complete sectioning of the SLL, we found significant lunate extension during wrist extension compared with the intact state while only nearsignificant differences during wrist flexion. Scaphoid motion was relatively unchanged but adopted a slightly more flexed position in wrist flexion. Additionally, there was a noteworthy increase in SL intercarpal motion and was most apparent in wrist flexion. The larger magnitude in angular changes in carpal rotation and intercarpal motion following complete SLL destabilization supports the notion that the SLL is the primary stabilizer of the SL joint and that a complete SLL injury is required before significant motion changes are seen in either the lunate or scaphoid. ${ }^{2,11-14}$ This study is partly in agreement with previously completed in vitro biomechanical studies showing that the complete sectioning of the SLL resulted in increased scaphoid flexion and lunate extension during wrist flexion-extension motion. ${ }^{12-14,23}$ 
Table 1 Mean angular change observed between the sectioning stages for wrist extension and flexion motion paths

\begin{tabular}{|c|c|c|c|c|c|c|}
\hline \multicolumn{7}{|c|}{ Wrist extension } \\
\hline & \multicolumn{2}{|l|}{ Lunate motion } & \multicolumn{2}{|l|}{ Scaphoid motion } & \multicolumn{2}{|l|}{ Scapholunate motion } \\
\hline & $\begin{array}{l}\text { Mean } \\
\text { difference (degrees) }\end{array}$ & Significance & $\begin{array}{l}\text { Mean } \\
\text { difference (degrees) }\end{array}$ & Significance & $\begin{array}{l}\text { Mean } \\
\text { difference (degrees) }\end{array}$ & Significance \\
\hline $\mathrm{S} 1 \rightarrow \mathrm{S} 2$ & 1.4 & 0.23 & 0.7 & 1 & -0.67 & 1 \\
\hline $\mathrm{S} 1 \rightarrow \mathrm{S} 3$ & 4 & 0.04 & 1 & 1 & -3.1 & 0.51 \\
\hline $\mathrm{S} 1 \rightarrow \mathrm{S} 4$ & 4.4 & 0.02 & -0.18 & 1 & -3.6 & 0.17 \\
\hline $\mathrm{S} 1 \rightarrow \mathrm{S} 5$ & 5.3 & 0.01 & 0.86 & 1 & -4 & 0.27 \\
\hline $\mathrm{S} 2 \rightarrow \mathrm{S} 3$ & 2.6 & 0.02 & 0.28 & 1 & -2.5 & 1 \\
\hline $\mathrm{S} 2 \rightarrow \mathrm{S} 4$ & 2.9 & 0.01 & -0.9 & 1 & -3 & 0.36 \\
\hline $\mathrm{S} 2 \rightarrow \mathrm{S} 5$ & 3.8 & 0.01 & 0.14 & 1 & -3.4 & 0.59 \\
\hline $\mathrm{S} 3 \rightarrow \mathrm{S} 4$ & 0.32 & 0.18 & -1.17 & 1 & -0.53 & 1 \\
\hline $\mathrm{S} 3 \rightarrow \mathrm{S} 5$ & 1.2 & 0.03 & -0.14 & 1 & -0.91 & 1 \\
\hline $\mathrm{S} 4 \rightarrow \mathrm{S} 5$ & 0.9 & 0.04 & 1 & 1 & -0.38 & 1 \\
\hline \multicolumn{7}{|c|}{ Wrist flexion } \\
\hline & \multicolumn{2}{|l|}{ Lunate motion } & \multicolumn{2}{|l|}{ Scaphoid motion } & \multicolumn{2}{|l|}{ Scapholunate motion } \\
\hline & $\begin{array}{l}\text { Mean } \\
\text { difference (degrees) }\end{array}$ & Significance & $\begin{array}{l}\text { Mean } \\
\text { difference (degrees) }\end{array}$ & Significance & $\begin{array}{l}\text { Mean } \\
\text { difference (degrees) }\end{array}$ & Significance \\
\hline $\mathrm{S} 1 \rightarrow \mathrm{S} 2$ & 1 & 1 & -0.82 & 0.45 & -1.8 & 0.35 \\
\hline $\mathrm{S} 1 \rightarrow \mathrm{S} 3$ & 4.7 & 0.05 & -3.5 & 0.08 & -8.2 & 0.03 \\
\hline $\mathrm{S} 1 \rightarrow \mathrm{S} 4$ & 5.5 & 0.03 & -3.3 & 0.18 & -8.8 & 0.05 \\
\hline $\mathrm{S} 1 \rightarrow \mathrm{S} 5$ & 6.7 & 0.02 & -3.3 & 0.26 & -10 & 0.03 \\
\hline $\mathrm{S} 2 \rightarrow \mathrm{S} 3$ & 3.7 & 0.16 & -2.7 & 0.15 & -6.4 & 0.09 \\
\hline $\mathrm{S} 2 \rightarrow \mathrm{S} 4$ & 4.5 & 0.08 & -2.5 & 0.41 & -7 & 0.12 \\
\hline $\mathrm{S} 2 \rightarrow \mathrm{S} 5$ & 5.7 & 0.03 & -2.5 & 0.61 & -8.1 & 0.09 \\
\hline $\mathrm{S} 3 \rightarrow \mathrm{S} 4$ & 0.84 & 0.27 & 0.24 & 1 & -0.58 & 1 \\
\hline $\mathrm{S3} \rightarrow \mathrm{S} 5$ & 2 & 0.07 & 0.28 & 1 & $\begin{array}{l}-1.7 \\
\end{array}$ & 0.88 \\
\hline $\mathrm{S} 4 \rightarrow \mathrm{S} 5$ & 1.2 & 0.12 & 0.04 & 1 & -1.1 & 0.33 \\
\hline
\end{tabular}

Note: The values in bold indicate that the values were significant at $p<0.05$.

Our results, however, suggest that the SLL plays a more critical role in lunate stability during wrist flexion.

After the additional sectioning of the STT and RSC ligaments, the carpals underwent further postural changes during wrist flexion and extension, with larger changes occurring in wrist flexion. Scaphoid motion was nonsignificantly altered, whereas the lunate progressed to an even more extended posture in wrist flexion and extension ( - Fig. 3). Our results contrast the study completed by Short et al, ${ }^{12}$ who reported that the sectioning of the STT and RSC ligaments following the SLL caused no further changes in SL kinematics during wrist flexion and extension. Our results support the notion that the STT and RSC ligaments are secondary stabilizing structures to the SL articulation and do, in fact, cause further deviation from normal SL kinematics following injury. Although the angular changes were small, we postulate that these small changes will be magnified in chronic injury states, thus making the results an interesting clinical finding. The absence of scaphoid instability is interesting because we observed noteworthy scaphoid mobility when the wrist was unloaded. It is reason- able to hypothesize that the compressive force within the wrist and the bony constraints of the radioscaphoid articulation stabilize the scaphoid.

Reconstructions including both the volar and dorsal portions of the SLL, STT, and RSC may be required as our results indicate that these structures play an important role in SL stability. It is possible that previous reconstruction techniques have unpredictable and/or poor results because they only reconstruct the dorsal or central portions of the SLL. Perhaps, if the entire SLL and secondary stabilizers were addressed, reconstruction techniques would produce more favorable clinical outcomes.

This study has several limitations. Wrist motion was simulated in planar flexion and extension, with mean angular differences in carpal kinematics reported in a single plane, thus not accounting for the complex multiplanar motion of the wrist. Additionally, although wrist motion was performed actively and loads were applied within a physiological range, ${ }^{26}$ this remains an estimation of in vivo loading scenarios. In addition, randomization of the sectioning protocol was not 


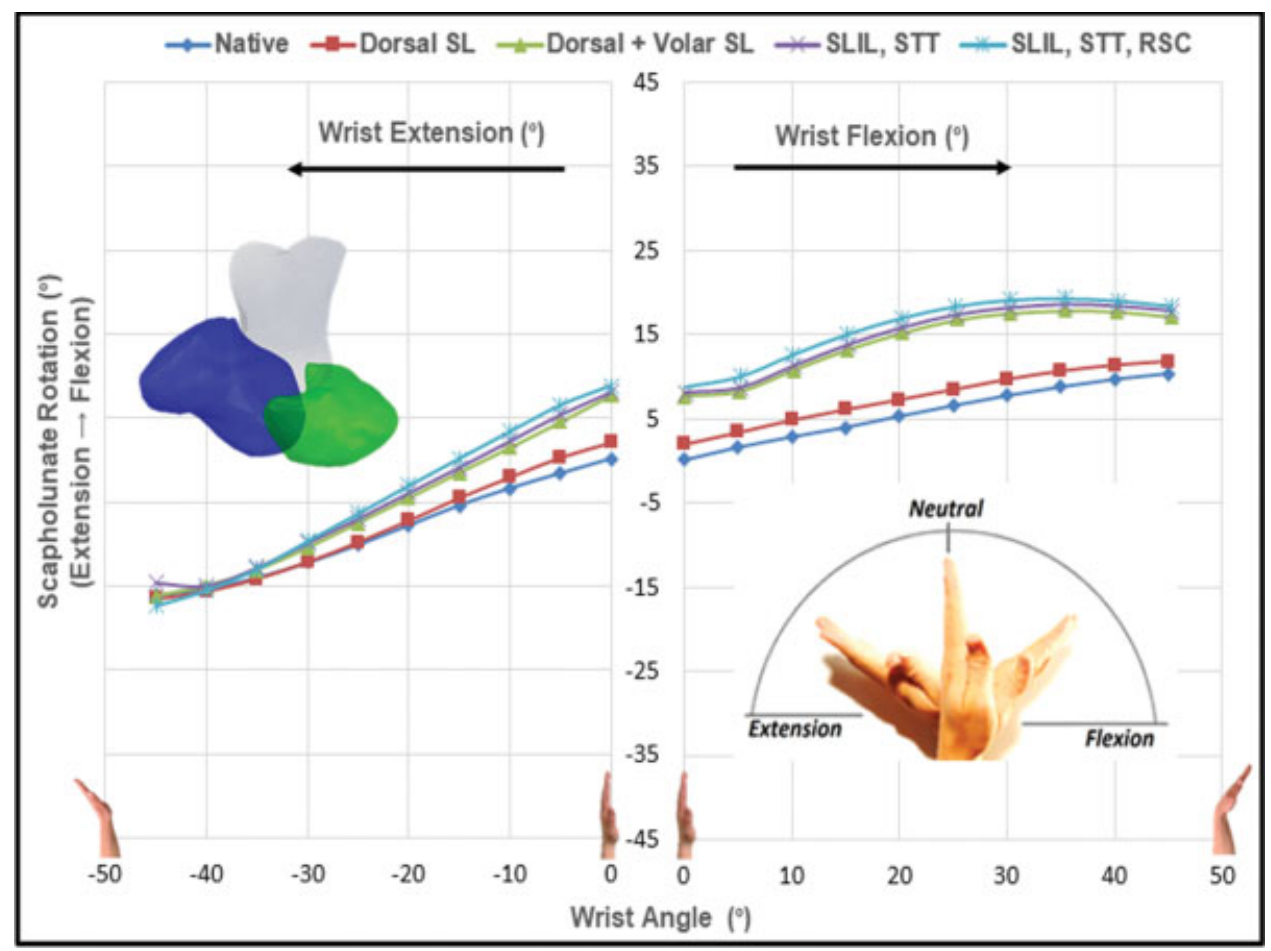

Fig. 5 Mean scapholunate motion in the flexion-extension plane for the flexion and extension motion paths. Sequential sectioning caused the scaphoid to adopt a more flexed position with respect to the lunate; these changes were largest in wrist flexion. Standard deviations were omitted for clarity (S1: 0.49-8.3; S2: 2-7.9; S3: 4.4-8.8; S4: 4.6-9.7; S5: 5.2-9.7).

studied due to the limitation of specimen availability. Previous biomechanical studies, which cycled the wrist $(\sim 1,000 \times)$, reported greater angular changes. ${ }^{27-29}$ Although we did not intentionally perform a cycling protocol, each specimen was subject to on average $178 \pm 32$ wrist flexion-extension motion trials. Finally, this study was limited to investigating the role of only two of the secondary stabilizers of the SL articulation. Future studies should investigate multiplanar carpal motion during complex wrist motions, as well as the randomization of ligament sectioning.

Overall, the current biomechanical study supports the hypothesis that the SLL is the primary stabilizer of the SL articulation and that the STT and RSC are secondary ligamentous restraints. The largest changes to carpal kinematics and intercarpal motion following sectioning were seen in wrist flexion, which is similar to observations from previous studies. ${ }^{14}$ Furthermore, our findings suggest that there are additional secondary soft tissue restraints that play a role in the stability and maintenance of normal SL kinematics. We propose addressing secondary stabilizers, such as the STT and RSC, during repair or reconstruction to achieve carpal stability, particularly in the setting of a complete SLL tear.

\section{Funding}

Dr. Johnson reports grants from the Canadian Institutes of Health Research Program Grant during the conduct of the study.

\section{Conflict of Interest}

None declared.

\section{References}

1 Wolfe SW, Neu C, Crisco JJ. In vivo scaphoid, lunate, and capitate kinematics in flexion and in extension. J Hand Surg Am 2000;25 (05):860-869

2 Kitay A, Wolfe SW. Scapholunate instability: current concepts in diagnosis and management.J Hand Surg Am 2012;37(10):2175-2196

3 Johnson JE, Lee P, McIff TE, Toby EB, Fischer KJ. Effectiveness of surgical reconstruction to restore radiocarpal joint mechanics after scapholunate ligament injury: an in vivo modeling study. J Biomech 2013;46(09):1548-1553

4 Werner FW, Dimitris C, Joyce DA, Harley BJ. Force in the scapholunate interosseous ligament during physiological wrist loading. J Hand Surg Am 2015;40(08):1-9

5 Kuo CE, Wolfe SW. Scapholunate instability: current concepts in diagnosis and management. J Hand Surg Am 2008;33(06): 998-1013

6 Ruby LK, An KN, Linscheid RL, Cooney WP III, Chao EYS. The effect of scapholunate ligament section on scapholunate motion. J Hand Surg Am 1987;12(5 Pt 1):767-771

7 O'Meeghan CJ, Stuart W, Mamo V, Stanley JK, Trail IA. The natural history of an untreated isolated scapholunate interosseus ligament injury. J Hand Surg Br 2003;28(04):307-310

8 Cooney WIII, ed The Wrist: Diagnosis and Operative Treatment. In: Kluwer W, ed. Vol. 8. 2nd ed. Rochester, MN: Wolters Kluwer; 2011

9 Berger RA. The gross and histologic anatomy of the scapholunate interosseous ligament. J Hand Surg Am 1996;21(02):170-178

10 Berger RA, Imeada T, Berglund L, An KN. Constraint and material properties of the subregions of the scapholunate interosseous ligament. J Hand Surg Am 1999;24(05):953-962

11 Short WH, Werner FW, Green JK, Masaoka S. Biomechanical evaluation of the ligamentous stabilizers of the scaphoid and lunate: part II. J Hand Surg Am 2005;30(01):24-34

12 Short WH, Werner FW, Green JK, Masaoka S. Biomechanical evaluation of ligamentous stabilizers of the scaphoid and lunate. J Hand Surg Am 2002;27(06):991-1002 
13 Short WH, Werner FW, Fortino MD, Palmer AK, Mann KA. A dynamic biomechanical study of scapholunate ligament sectioning. J Hand Surg Am 1995;20(06):986-999

14 Short WH, Werner FW, Green JK, Sutton LG, Brutus JP. Biomechanical evaluation of the ligamentous stabilizers of the scaphoid and lunate: part III. J Hand Surg Am 2007;32(03):297-309

15 Waters MS, Werner FW, Haddad SF, McGrattan ML, Short WH. Biomechanical evaluation of scaphoid and lunate kinematics following selective sectioning of portions of the scapholunate interosseous ligament. J Hand Surg Am 2016;41(02):208-213

16 Lavernia CJ, Cohen MS, Taleisnik J. Treatment of scapholunate dissociation by ligamentous repair and capsulodesis. J Hand Surg Am 1992;17(02):354-359

17 Blatt G. Capsulodesis in reconstructive hand surgery. Dorsal capsulodesis for the unstable scaphoid and volar capsulodesis following excision of the distal ulna. Hand Clin 1987;3(01):81-102

18 Weiss A-P. Scapholunate ligament reconstruction using a boneretinaculum-bone autograft.J Hand Surg Am 1998;23(02):205-215

19 Watson HK, Weinzweig J, Guidera PM, Zeppieri J, Ashmead D. One thousand intercarpal arthrodeses. J Hand Surg Br 1999;24(03): 307-315

20 Kleinman WB. Long-term study of chronic scapho-lunate instability treated by scapho-trapezio-trapezoid arthrodesis. J Hand Surg Am 1989;14(03):429-445

21 Wyrick JD, Stern PJ, Kiefhaber TR. Motion-preserving procedures in the treatment of scapholunate advanced collapse wrist: proximal row carpectomy versus four-corner arthrodesis. J Hand Surg Am 1995;20(06):965-970
22 Wyrick JD, Youse BD, Kiefhaber TR. Scapholunate ligament repair and capsulodesis for the treatment of static scapholunate dissociation. J Hand Surg Br 1998;23(06):776-780

23 Werner FW, Short WH, Green JK. Changes in patterns of scaphoid and lunate motion during functional arcs of wrist motion induced by ligament division. J Hand Surg Am 2005;30(06):1156-1160

24 Iglesias D. (2015). Development of an In-Vitro Passive and Active Motion Simulator for the Investigation of Wrist Function and Kinematics. Electronic Thesis and Dissertation Repository, University of Western Ontario. http://ir.lib.uwo.ca/cgi/viewcontent. cgi? article $=3380$ \& context $=$ etd.

$25 \mathrm{Wu}$ G, van der Helm FCT, Veeger HEJ, et al; International Society of Biomechanics. ISB recommendation on definitions of joint coordinate systems of various joints for the reporting of human joint motion-part II: shoulder, elbow, wrist and hand. J Biomech 2005; 38(05):981-992

26 Werner FW, Palmer AK, Somerset JH, et al. Wrist joint motion simulator. J Orthop Res 1996;14(04):639-646

27 Pollock PJ, Sieg RN, Baechler MF, Scher D, Zimmerman NB, Dubin $\mathrm{NH}$. Radiographic evaluation of the modified Brunelli technique versus the Blatt capsulodesis for scapholunate dissociation in a cadaver model. J Hand Surg Am 2010;35(10):1589-1598

28 Lee SK, Zlotolow DA, Sapienza A, Karia R, Yao J. Biomechanical comparison of 3 methods of scapholunate ligament reconstruction. J Hand Surg Am 2014;39(04):643-650

29 Short WH, Werner FW, Sutton LG. Treatment of scapholunate dissociation with a bioresorbable polymer plate: a biomechanical study. J Hand Surg Am 2008;33(05):643-649 